\title{
Retinal Function in Best Macular Dystrophy: Relationship between Electrophysiological, Psychophysical, and Structural Measures of Damage
}

\author{
Susana Maia-Lopes, ${ }^{1}$ Eduardo Duarte Silva,,${ }^{1,2}$ Aldina Reis, ${ }^{1,2}$ Maria Fátima Silva, ${ }^{1}$ \\ Catarina Mateus, ${ }^{1,2}$ and Miguel Castelo-Branco ${ }^{1}$
}

\begin{abstract}
Purpose. To establish structure-function correlations across the visual field, to investigate disease progression in Best macular dystrophy (BMD), by correlating structural damage with retinal function as assessed by the combination of psychophysics and multifocal electrophysiology.
\end{abstract}

Methods. Spatial achromatic and chromatic contrast sensitivities (probing red-green and blue-yellow pathways) were assessed using custom-made psychophysical software to evaluate retinal damage in BMD and age-matched control eyes $(n=19$ and $n=22$, respectively). Neurosensory retinal dysfunction was also evaluated by means of multifocal electroretinography (mfERG). Correlation analysis was performed between functional parameters in BMD, clinical measures, and morphologic data obtained by optical coherence tomography (OCT).

Results. Significant peripheral impairment of retinal function, as measured by mfERG and spatial achromatic contrast sensitivity (CS) methods, was found in BMD. Furthermore, changes in thickness of the neurosensory retina, as measured by OCT, and reduced mfERG responses were also indicators of early loss in BMD and often occurred even with preserved visual acuity. Disease duration was significantly correlated with psychophysical deterioration in chromatic and achromatic tasks but not with mfERG measures. Interestingly, partial correlation analysis revealed a significant independent correlation with our CS measures.

Conclusions. Novel topographic achromatic and chromatic CS methods can detect and quantify functional impairment in early stages of BMD, including the involvement of the peripheral retina and the central chromatic pathway, and can provide new pathophysiological information with added value in relation to electrophysiological and structural measures of damage. (Invest Ophthalmol Vis Sci. 2008;49:5553-5560) DOI:10.1167/ iovs.08-2093

From the ${ }^{1}$ Visual Neuroscience Laboratory, IBILI, Faculty of Medicine, University of Coimbra, Coimbra, Portugal; and the ${ }^{2}$ University Hospital of Coimbra, Coimbra, Portugal.

Supported by Portuguese Foundation for Science and Technology Grants POCI-SAU-OBS-57070-2004 and POCI-SAU-OBS-60281-2004; the Gulbenkian Foundation (Biomarkers of Ageing Project); and European Network Grant EVI-GENORET LSHG-CT-2005-512036. SM-L and MFS were supported by individual fellowships (SFRH/BD/11828/2003 and $\mathrm{SFRH} / \mathrm{BD} / 18777 / 2004$, respectively) from the Portuguese Foundation for Science and Technology

Submitted for publication March 29, 2008; revised July 24, 2008; accepted October 13, 2008.

Disclosure: S. Maia-Lopes, None; E. Duarte Silva, None; A. Reis, None; M.F. Silva, None; C. Mateus, None; M. Castelo-Branco, None The publication costs of this article were defrayed in part by page charge payment. This article must therefore be marked "advertisement" in accordance with 18 U.S.C. $\$ 1734$ solely to indicate this fact.

Corresponding author: Miguel Castelo-Branco, Visual Neuroscience Laboratory, IBILI, Faculty of Medicine, University of Coimbra, Az. de Sta Coimba, 3000-354 Coimbra, Portugal; mcbranco@ibili.uc.pt.
$\mathrm{B}$ est vitelliform macular dystrophy (BMD), also known as vitelliform macular degeneration type 2 , is an autosomal dominant disease that affects the central retina and is characterized by typical egg-yolk macular lesions caused by accumulations of lipofuscin within and beneath the retinal pigment epithelium (RPE). ${ }^{1,2}$ Atrophy of RPE in the fovea usually affects the overlying photoreceptors and leads to the impairment of central visual function. The finding of abnormal EOG results with normal standard ERG results is a hallmark of BMD, supporting the notion that the primary defect derives from the RPE. Several reports have shown significant variable clinical intrafamilial and interfamilial expressivity and expressivity across the eyes of affected persons. ${ }^{3-5}$

Mutations in the BMD gene, termed Best1 (formerly VMD2; MIM 153700) and mapped to 11q13, have been identified in affected families. ${ }^{6,7}$ Best 1 codes for a 585-amino acid transmembrane peptide with a predicted size of $68 \mathrm{kDa}$, named bestrophin. Best 1 mRNA is expressed mainly in the retina, especially in the basolateral plasma membrane of retinal pigment epithelial cells. ${ }^{8}$ In 2002, Sun et al. ${ }^{9}$ demonstrated that the bestrophin homologs are oligomeric chloride channels sensitive to intracellular calcium. However, no evidence for direct involvement of bestrophin in the generation of light peak was found by Marmorstein et al. ${ }^{10}$ in 2004.

The pathophysiology of BMD is not yet clear, and it may well be necessary to revisit the hypothesis that photoreceptor damage may occur even earlier in the natural history of the disease than previously believed. Indeed, in donor eyes from BMD patients, retinal pigment epithelial and foveal photoreceptor loss was observed. ${ }^{11}$ In 1982 , Frangieh et al. ${ }^{12}$ found fine granular deposits in degenerating photoreceptors and in Müller cells, providing further evidence that the neural retina is an important locus of damage.

Recent findings have indeed shown that cone function is impaired in the very early stage of the disease; hence, it cannot be excluded that a disease mechanism operates early at the photoreceptor level. Abnormal flicker fusion threshold intensities and chromatic dysfunction have been observed in BMD patients with normal and near-normal Snellen visual acuity. ${ }^{13,14}$ Furthermore, L/M-cone driven ERG showed that these two cone pathways are differentially affected by retinal degeneration. ${ }^{15}$ Few studies, however, have focused on the quantification of spatial retinal impairment in BMD and its correlation with disease progression and visual acuity (VA). Macular dysfunction was previously assessed by Falsini et al., ${ }^{16}$ who recorded reduced macular flicker ERGs in patients with earlystage BMD. We previously demonstrated abnormal foveomacular chromatic contrast sensitivity (CS) along all the three cone confusion lines in BMD, even when VA was still spared. ${ }^{14} \mathrm{~A}$ recent study has documented reduced multifocal electroretinography (mfERG) amplitudes in BMD, in the centralmost rings, and in a stage-dependent manner. ${ }^{17}$

It is important to extend these results using novel spatial vision phenotyping methods, namely topographic CS measurements that can shed light on peripheral impairment and can 
TABLE 1. Clinical Characteristics of BMD Patients

\begin{tabular}{|c|c|c|c|c|c|c|c|c|}
\hline \multirow[b]{2}{*}{ Family } & \multirow[b]{2}{*}{ Subject } & \multirow[b]{2}{*}{ Sex } & \multirow[b]{2}{*}{$\begin{array}{l}\text { Age } \\
\text { (y) }\end{array}$} & \multirow{2}{*}{$\begin{array}{c}\text { Onset } \\
(y)\end{array}$} & \multirow[b]{2}{*}{$\begin{array}{c}\text { Duration } \\
\text { (y) }\end{array}$} & \multicolumn{2}{|c|}{ VA } & \multirow{2}{*}{$\begin{array}{l}\text { Color } \\
\text { Vision }\end{array}$} \\
\hline & & & & & & OD & OS & \\
\hline \multirow[t]{2}{*}{ I } & 1 & M & 36 & 16 & 20 & 0.8 & 0.8 & Normal \\
\hline & 2 & $\mathrm{~F}$ & 10 & 8 & 2 & 0.8 & 0.8 & Normal \\
\hline \multirow[t]{2}{*}{ II } & 3 & M & 51 & 16 & 35 & 1.0 & 1.0 & $P+T$ \\
\hline & 4 & M & 19 & 6 & 13 & 1.0 & 0.9 & $\mathrm{~T}$ \\
\hline \multirow[t]{2}{*}{ III } & 5 & M & 37 & 25 & 12 & 1.0 & 0.1 & $\mathrm{P}$ \\
\hline & 6 & M & 35 & 23 & 12 & 0.2 & 0.2 & $P+T$ \\
\hline \multirow[t]{3}{*}{ IV } & 7 & M & 14 & 6 & 8 & 0.2 & 0.8 & $P+T$ \\
\hline & 8 & M & 53 & 22 & 31 & 0.8 & 0.8 & $P+T$ \\
\hline & 9 & $\mathrm{~F}$ & 25 & 18 & 8 & 0.8 & 0.8 & $P+T$ \\
\hline V & 10 & M & 58 & 40 & 18 & 0.8 & 0.6 & $\mathrm{~T}$ \\
\hline VI & 11 & M & 42 & 33 & 9 & 1.0 & 1.0 & $\mathrm{~T}$ \\
\hline \multirow[t]{7}{*}{ VII } & 12 & $\mathrm{~F}$ & 44 & 10 & 34 & 0.4 & 0.3 & $\mathrm{P}+\mathrm{T}$ \\
\hline & 13 & M & 20 & 5 & 15 & 1.0 & 1.0 & $\mathrm{~T}$ \\
\hline & 14 & M & 14 & 6 & 8 & 1.0 & 0.2 & $P+T$ \\
\hline & 15 & M & 20 & 5 & 15 & 0.2 & 0.4 & $\mathrm{~T}$ \\
\hline & 16 & M & 45 & 10 & 35 & 0.3 & 0.9 & $P+T$ \\
\hline & 17 & F & 27 & 26 & 1 & 0.8 & 1.0 & $P+T$ \\
\hline & 18 & M & 36 & 15 & 21 & 0.4 & 0.8 & $P+T$ \\
\hline VIII & 19 & $\mathrm{~F}$ & 14 & 11 & 3 & 0.3 & $<0.1$ & ND \\
\hline
\end{tabular}

Age, onset (age at the first detected clinical manifestation), disease duration and best-corrected visual acuities for right (OD) and left (OS) eyes are indicated. Color vision function was measured using an anomaloscope and was classified according to protanomaly (P), deutanomaly (D), and tritanomaly (T). ND, not determined.

serve as potential biomarkers to evaluate disease progression. Accordingly, the present study aimed to evaluate the relevance of novel spatial achromatic and chromatic CS methods in phenotyping BMD and their correlation with functional (mfERG), morphologic (optical coherence tomography [OCT]), and clinical measures.

\section{Methods}

\section{Characterization of the Clinical Group}

Nineteen BMD patients ( $n=19$ eyes; mean age, 31.9 years; range, $10-58$ years) from eight families were included in the study and were compared with an age-similar group of 22 normal-sighted controls ( $n=$ 22 eyes; mean age, 29 years; range, 9-58 years). Analysis of variance showed no significant age difference between groups (F-test, $P=$ $0.445)$.

The diagnosis of BMD was based on the presence of the typical macular vitelliform lesion assessed by fundus photography, reduced light-peak to dark-trough ratio of the EOG (Arden ratio lower than 1.8), and family history (only 3 of 19 had no obvious dominant inheritance and were, therefore, classified as sporadic). Fundus photographs were taken according to the guidelines of the Fundus Photograph Reading Center Department of Ophthalmology and Visual Sciences of the University of Wisconsin. Best-corrected visual acuity was obtained by postcycloplegic manifest refraction on Snellen charts. No other ophthalmologic or systemic condition was present. Clinical data of BMD patients are shown in Table 1. Disease duration was defined as the period since decreased VA was first noted and was chosen as a quantitative clinical parameter for correlation analyses. One eye per patient was studied. We chose the eye with best VA ( VA $\geq 0.2)$; if both eyes had similar VA, the right eye was selected for further analysis. Color vision function was measured with the use of an anomaloscope (model IF-2; Roland Consult, Wiesbaden, Germany) as the clinical gold standard and with Rayleigh and Moreland equations and thus was classified in protanomaly, deutanomaly, and tritanomaly classes.

Informed consent was obtained from all subjects, in strict accordance with the institutional guidelines of our local ethics committee and after explanation of the objectives and possible consequences of the study. The research was conducted in accordance with the tenets of the Declaration of Helsinki.

\section{Contrast Sensitivity Psychophysical Tasks}

Achromatic Contrast Sensitivity. CS was assessed independently for each random location (one central, four paracentral, four peripheral; Fig. 1) in an interleaved manner. Grating stimuli (static, 3.5 cpd) were generated from a CRS/VSG 2/5 graphics card (Cambridge Research Systems [CRS], Rochester, UK), and displayed on a 21-inch gamma-corrected monitor (frame rate $100 \mathrm{~Hz}$; Trinitron GDM-F520; Sony, Tokyo, Japan) with a viewing distance of $36 \mathrm{~cm}$ and under a mean background luminance of $51 \mathrm{~cd} / \mathrm{m}^{2}$ (Fig. 1).

Luminance contrast or modulation of the stimulus was expressed according to Michelson: luminance contrast $(\%)=100 \times\left(L_{\max }-\right.$ $\left.L_{\text {min }}\right) /\left(L_{\max }+L_{\min }\right)$. An adaptive logarithmic staircase strategy was used to obtain psychophysical thresholds. Staircases were run for a total of four reversals, with the contrast at the final two reversals averaged to estimate the contrast threshold. Results were expressed in terms of decibel $(\mathrm{dB})$ units: $\mathrm{dB}=20 \times \log (1 / c)$, with contrast $c$ measured as a percentage.

This spatial testing procedure was performed monocularly. Subjects were instructed to fixate a black square $\left(1^{\circ} \times 1^{\circ}\right)$ in the center of the screen and to report the presence of vertical "striped" targets (detection task) by means of a button press. Stimulus duration was 200 ms, and ISI was jittered between 2300 to 2800 seconds. Participant reliability was evaluated by the inclusion of false-positive and -negative "catch trials," and all results with false-positive and false-negative errors of $33 \%$ or greater were excluded, according to standard criteria. ${ }^{18}$ Fixation loss was monitored using an eye-tracking methodology (CRS device) that provided detailed measurements of pupil position. Participants with difficulties in fixation were excluded.

For the analyses of the CS in eccentricity, three zones were defined: zone 0 corresponded to the $5^{\circ}$ central visual field, zone 1 contained locations between $5^{\circ}$ and $10^{\circ}$ eccentricity, and zone 2 included stimuli between $10^{\circ}$ and $20^{\circ}$ eccentricity.

Chromatic Contrast Sensitivity. To evaluate independently red-green and blue-yellow pathways, we used sinusoidal gratings similar to the achromatic stimulus (static, $3.5 \mathrm{cpd}$ ) modulated along lines 
Figure 1. Tested locations and stimulus layout for the achromatic CS test. CS was assessed independently and in an interleaved manner for each of the nine locations tested.

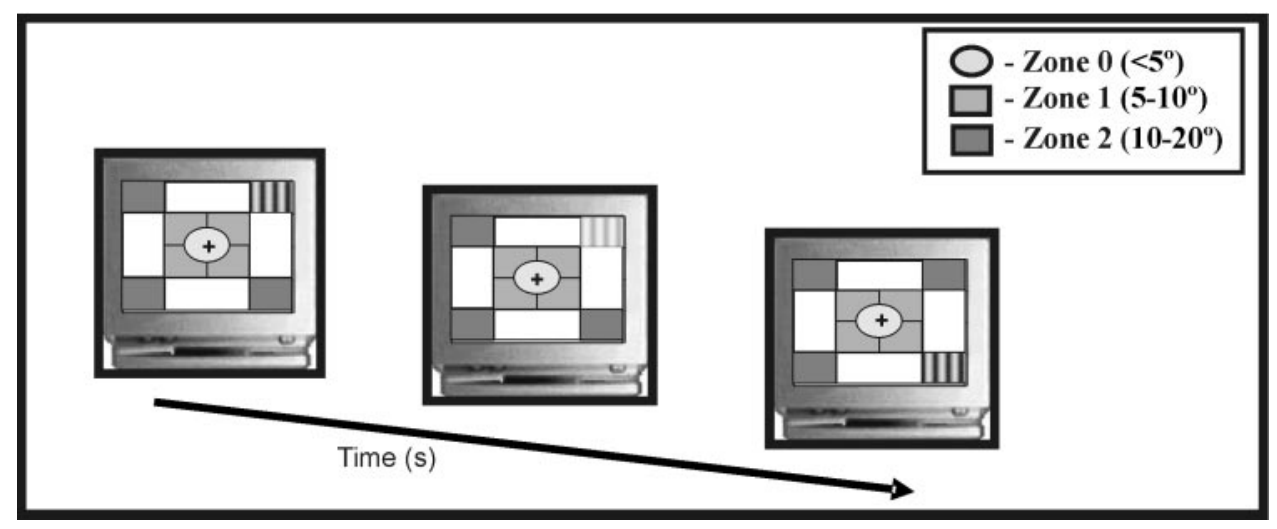

in color space close the three main confusion axes within the Commission International d'Eclairage (CIE) $1931 x, y$ space $^{14,19}$ but with slight offsets from the isoluminant plane to ensure similar computed quantal catches across tasks. This allowed for comparison with our own previous study, ${ }^{14}$ in which isoluminance was prioritized over quantal catch equivalence. We used the same setup for stimuli generation and monitor calibration, staircase procedure, viewing distance, subject instructions, and performance reliability as defined for the achromatic CS tests, which ensured test comparability.

We measured chromatic contrast thresholds in five locations within $10^{\circ}$ of visual field (a central circular test field subtending $2^{\circ}$ of visual angle, zone 0 ; and four $5^{\circ}-10^{\circ}$ testing locations, zone 1 ; displayed as shown in Fig. 2). Psychophysical chromatic sensitivity was tested in a randomly interleaved manner for each contrast axis. The red-green pathway was tested with two distinct procedures by modulating contrast close to the protan and deutan axes (averages across these two were used as an estimate of red-green CS). Staircase vector extreme coordinates in the CIE $1931 x, y$ color space were as follows: protan, $x=0.433, y=0.295$; deutan, $x=0.371, y=0.243$. The blue-yellow stimulus was modulated close to the tritan axis (extreme CIE $1931 x, y$ chromaticity coordinate $x=0.254, y=0.197$ ). The white starting point was $x=0.306, y=0.314$. Mean background luminance and stimulus luminance were set equally to $22.20,41.50$, and $6.75 \mathrm{~cd} / \mathrm{m}^{2}$ for protan, deutan, and tritan axes, respectively, to obtain nearly identical average cone quantal catches at $2^{\circ}$ and $10^{\circ}(9.79 \pm 0.64$ [SD] $\log$ quanta $\cdot \mathrm{s}^{-1} \cdot$ cone $^{-1}$ for $2^{\circ}$ stimulus and $9.82 \pm 0.66[\mathrm{SD}] \log$ quanta $\cdot \mathrm{s}^{-1} \cdot$ cone $^{-1}$ for $10^{\circ}$ ). Therefore, chromatic pathways ${ }^{20,21}$ could be compared at approximately the same adapting level. The effective quantal catch produced in each cone by each primary was calculated by multiplying the Stockman and Sharpe cone fundamentals for $2^{\circ}$ or $10^{\circ}$ with the emission spectra of the three phosphors and a constant, $k$, and by integrating the product over wavelength. ${ }^{22,23}$ The constant, $k$, is different for each cone, depending on the product of the ocular media transmittance and the absolute absorption coefficients for the wavelength of the maximal absorption probability for each cone. The emission spectra of the red, green, and blue phosphors of the monitor were measured separately in steps of $1 \mathrm{~nm}$ with a calibrated spectroradiometer (CRS SpectroCAL, Rochester, UK).

\section{Electrophysiological Recordings}

We recorded mfERGs using DTL fiber electrodes, after a light adaptation period of 10 minutes and pupil dilation with tropicamide, before fundus photography, with a commercial system (RETIscan System; Roland Consult). ${ }^{24}$ Refractive errors were corrected in relation to the viewing distance. The stimulus used in the mfERG consisted of 61 hexagons covering a visual field of up to $30^{\circ}$ and presented on a 20 -inch monitor at a viewing distance of $33 \mathrm{~cm}$. Luminance was 120 $\mathrm{cd} / \mathrm{m}^{2}$ for white hexagons and approximately $1 \mathrm{~cd} / \mathrm{m}^{2}$ for black hexagons, resulting in a Michelson contrast of $99 \%$. The hexagonal areas increased with eccentricity to compensate for local differences in signal amplitude because of differences in cone density across the retina (leading to a fourfold change in hexagon area size). Each hexagon was temporally modulated between light and dark according to a binary $\mathrm{m}$-sequence (frame rate, $60 \mathrm{~Hz}$ ). Observers were instructed to fixate a small black cross in the center of the stimulus. Fixation was continuously checked by means of online video-monitoring during the approximately 8-minute recording sessions. To improve fixation stability, sessions were broken into 47-second segments; eight trials were recorded in total. Signals were amplified with a gain of 100,000 and were band-pass filtered $(5-300 \mathrm{~Hz})$.

Reference and ground electrodes were attached to the ipsilateral outer canthus and forehead, respectively. The surface electrode im-
A

Figure 2. Tested locations and stimulus layout for the chromatic CS test (A) and the CIE $1931 x, y$ chromaticity diagram showing the white, protan, deutan, and tritan points (B). Note that luminance was manipulated such that cone quantal catches remained constant. CS obtained for each of the three color axes tested was expressed in decibels.

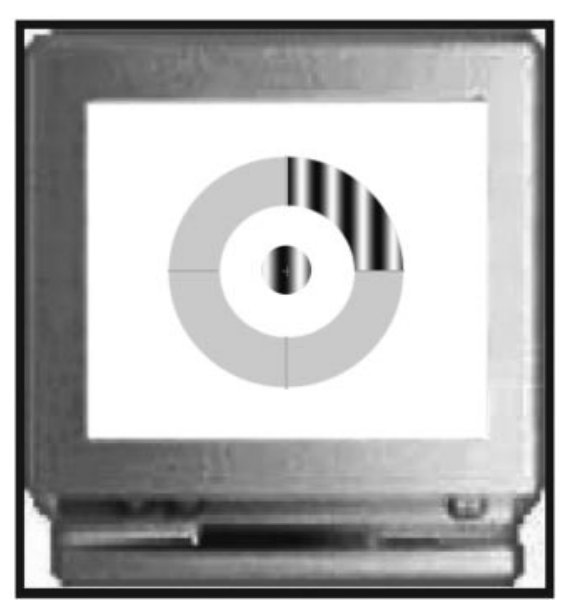

B

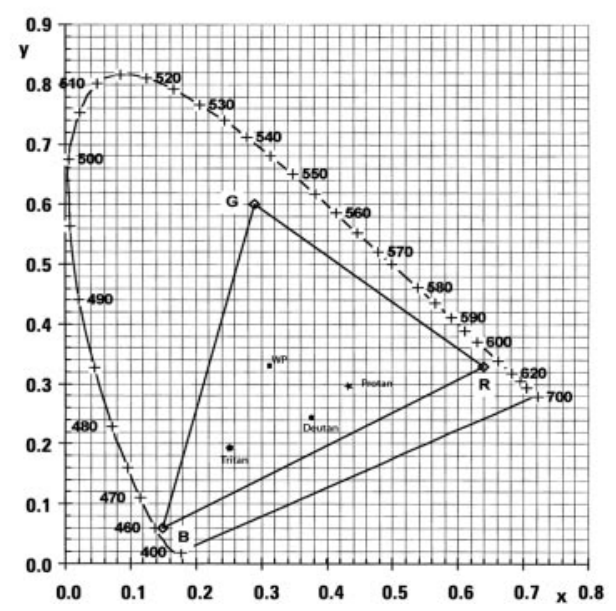


pedance was less than $10 \mathrm{k} \Omega$. Analyses were performed with the system software (RETIscan; Roland Consult) and standard statistical packages. First-order kernels were used for mfERG analysis because of their close correlation with the function of the outer retina. ${ }^{25}$ The obtained local ERGs responses were normalized by the area of stimulus delivery to obtain a density response (nv/ $\mathrm{deg}^{2}$ ). For each hexagon, the peak amplitude of P1-defined as the difference between N1 and P1 amplitudes-the N1 peak, and the implicit time of $\mathrm{P} 1$ component were computed. To easily evaluate spatial differences of the local ERG responses, responses from the 61 elements were divided into averages of five concentric rings around the fovea.

\section{Optical Coherence Tomography}

We used an OCT device (Stratus OCT; Carl Zeiss Meditec, Dublin, CA) to obtain cross-sectional images centered in the macula, ${ }^{26}$ with axial resolution of $10 \mu \mathrm{m}$ or less, transversal resolution of $20 \mu \mathrm{m}$, and longitudinal scan range of $2 \mathrm{~mm}$. With this OCT device (Stratus OCT; Carl Zeiss Meditec), six radial line scans $6 \mathrm{~mm}$ in length and 128 A-scans $30^{\circ}$ apart were scanned in 1.92 seconds, and a nine-region retinal thickness map was obtained by segmenting the retina from other layers with an algorithm detecting the edge of the RPE and the photoreceptor layer.

Macular retinal thickness was calculated by computing the distance between the signal from the vitreoretinal interface and the signal from the anterior boundary of the RPE. Retinal thickness was presented as a nine-region thickness map showing the interpolated thickness for each area, with a central circle of $500 \mu \mathrm{m}$ radius (ring 0 ) and two outer circles with radii of $1500 \mu \mathrm{m}$ (ring 1) and $3000 \mu \mathrm{m}$ (ring 2). The interpolated thickness was displayed using a false color scale, in which bright colors (red and white) corresponded to thickened areas and darker colors (blue and black) were assigned to thinner areas.

\section{Statistical Analysis}

We performed analysis of variance (ANOVA), with post hoc Fisher protected least significant difference (PLSD) correction using statistical software (StatView; Abacus Concepts, Berkeley, CA). Results with $P<$ 0.05 were considered statistically significant. Correlation analyses were performed using Fisher-to-Z transformation of Pearson coefficients. In cases of ranked variables, such as disease stage, correlation analyses were computed using the Spearman coefficient.

\section{RESUlts}

\section{Achromatic Contrast Sensitivity}

Representative maps of achromatic CS from BMD and control participants whose performance was close to their group median are illustrated in Figure 3A. These CS maps demonstrate significant impairment of spatial CS in BMD (Fig. 3B; $P<$ 0.0001 for the global grand average of CS; ANOVA). Comparison of CS across three eccentricity zones showed, as expected, higher CS for central locations (zone 0) and locations up to $10^{\circ}$ (zone 1) compared with the more peripheral tested locations (zone 2), in the aged-matched control group. This was also true for the BMD group. Further analysis confirmed the hypothesis that deterioration of CS function in BMD is not specifically confined to the centralmost regions of the visual field. Indeed, we have found significant impairment at all tested eccentricities $(P=0.0012, P=0.0001$, and $P<0.0001$ for zone 0 , zone 1 , and zone 2 , respectively; Fisher PLSD).

Considering individual data, we found that only 3 of the 19 affected eyes exhibited CS above the lower fifth percentile of the control group, corresponding to eyes with VA of 0.8 or better and with less than 9 years of disease progression.

We performed correlation analyses between CS and clinical measures to better understand patterns of disease progression. We found a significant correlation between the global grand
$\mathbf{A}$
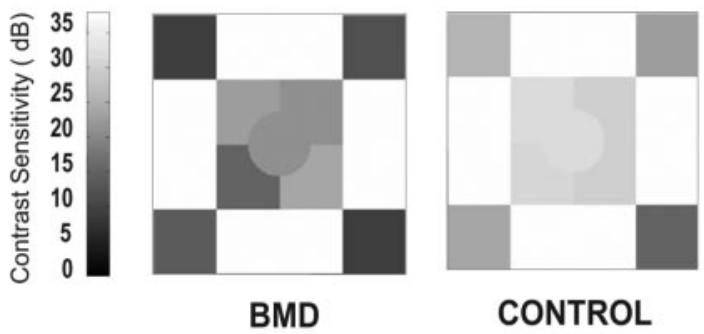

B

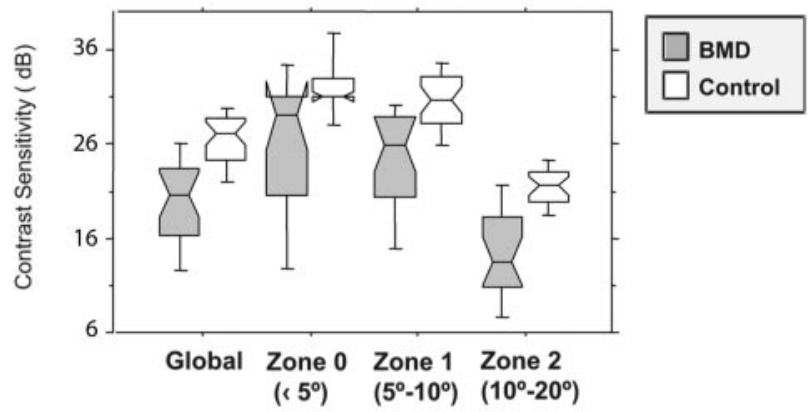

C

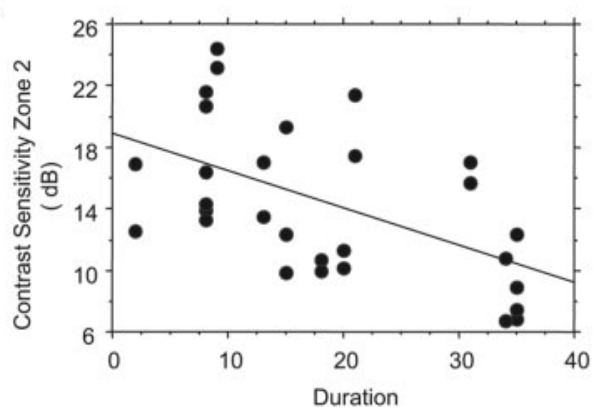

Zone $2=18,951-, 244$ * Duration; $\mathrm{R}^{\wedge} 2=, 285$

FIGURE 3. Achromatic CS function (ACS) in BMD patients. (A) Maps of ACS from representative BMD patients and control subjects. (B) Box plots of CS from the three different eccentricity tested regions. (C) Correlation of $\mathrm{CS}$ at $10^{\circ}$ to $20^{\circ}$ regions (zone 2) with disease duration.

average CS and disease stage (Rho correlation coefficient $[r]=$ $-0.504 ; P=0.027)$. Additional correlation analyses were undertaken to find the spatial source that best explained these results and revealed that this pattern was significant for the central regions, in contrast with the more peripheral locations ( $r=-0.507, P=0.025$ for zone $0 ; r=-0.517, P=0.022$ for zone 1 ; and $r=-0.378, P=0.111$ for zone 2). Interestingly, when we analyzed disease progression in terms of disease duration, an opposite spatial relation was observed, with significant correlations achieved in the more peripheral retina (Fig. 3C; $r=-0.219, P=0.373$ for zone $0 ; r=-0.246, P=$ $0.280 ; r=-0.534, P=0.017)$. These effects suggest that the impairment of CS in peripheral regions is clinically useful. Accordingly, CS measures obtained from more peripheral regions of the retina are potentially useful to monitor disease progression and duration. No significant correlation was found with VA (except, as expected for zone $0, r=0.472$ and $P=$ 0.040 ), further proving the dissociation with central visual function and the importance of other functional measures to evaluate disease progression. 
A

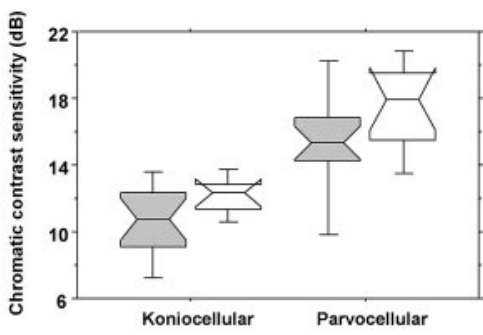

B

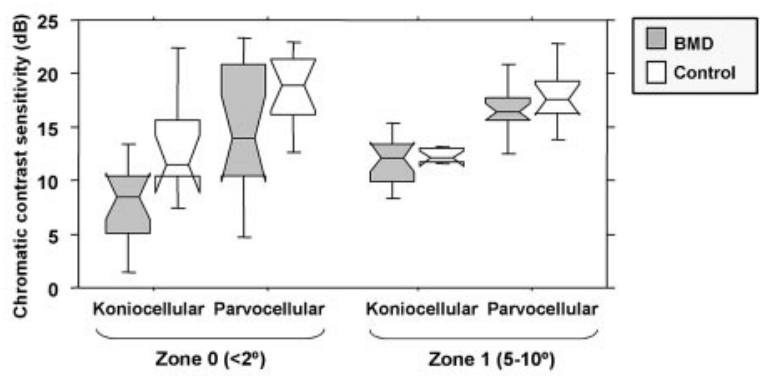

C
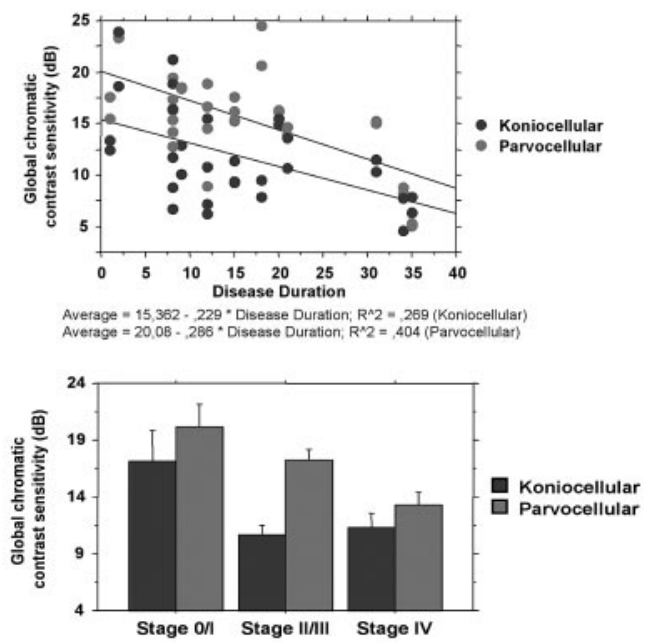

Figure 4. Chromatic CS function in BMD patients. (A) Bar plot of blue-yellow and red-green chromatic CS in BMD patients and control subjects. (B) Box plots of chromatic CS sorted across central (zone 0) and peripheral (zone 1) test locations. (C) Correlation of chromatic CS across distinct color pathways with disease duration and disease stage.

\section{Chromatic Contrast Sensitivity}

We determined the CS for red-green and blue-yellow chromatic pathways in BMD patients and control subjects. In patient and control groups, higher average CS was observed for the redgreen than for the blue-yellow pathways $(P=0.033$ and $P=$ 0.0001 for BMD patients and control subjects, respectively; Fig. 4A). Spatial disease-related dissociation in CS was again found, but with an opposite bias compared with the achromatic task and more evident central impairment. If only global measures were to be considered, significant differences between BMD patients and control subjects were found for grand average chromatic CS $(P=0.044)$ but not for each chromatic axis $(P=$ 0.092 and $P=0.155$ for blue-yellow and red-green biased pathways, respectively), suggesting that chromatic deficits are detected with smaller statistical power when isoquantal catches are ensured and isoluminance is broken (for an alternative approach, see Campos et al. ${ }^{14}$ ). When the analysis was performed separately for each test zone ( 0 and 1$)$, we observed an overall impairment of chromatic function in BMD patients in the most central zone for both tests (Fig. $4 \mathrm{~B}$; zone $0, P=$
0.011; zone $1, P=0.293$ ). However, post hoc analyses revealed that this effect was contributed primarily from impairment in the blue-yellow test (zone $0 ; P=0.013$ ). No significant dysfunction of chromatic CS function was found for the more peripheral locations in BMD patients (zone 1). These results suggest significant chromatic impairment (in agreement with our own previous study ${ }^{14}$ ), albeit with higher statistical power under isoluminant conditions compared with quantal catch equivalence.

For chromatic pathways, reductions of CS in BMD patients showed strong and significant correlations with disease progression, as assessed by analysis of the roles of disease duration and stage, as shown in Figure 4C (disease duration: $r=$ $-0.691, P=0.002$, for red-green performance measures; $r=$ $-0.562, P=0.022$, for blue-yellow performance measures). When considering correlation of chromatic CS with disease stage, we found a pattern of significant correlations for the centralmost areas (zone 0: Rho $r=-0.553, P=0.025$ for red-green axes; and Rho $r=-0.597, P=0.017$ for the blue-yellow pathway) but not for the more peripheral retina locations (zone 1: $r=-0.351, P=0.186$ for the red-green axes and $r=-0.216, P=0.295$ for the blue-yellow pathway). No significant correlations were found between visual acuity and impairment in any of the chromatic CS tests $(r=-0.456$ and $P=0.068$ for red-green axes; $r=-0.375$ and $P=0.134$ for blue-yellow pathway; Spearman correlation coefficients).

Electrophysiological Observations. mfERGs of a control subject and a BMD patient are shown in Figure 5A. There was a substantial reduction in mfERG amplitude responses in BMD patients when compared with controls (Fig. 5B; $P<0.0001$ for both $\mathrm{N} 1$ and $\mathrm{P} 1$ components). With regard to the N1 and P1 mfERG components, amplitudes were found to be dramatically reduced for all rings in BMD patients (mfERG responses for N1 amplitude rings $1-3$ and 5 and for $\mathrm{P} 1$ amplitude rings $1-5, P<$ 0.005 ; for $\mathrm{N} 1$ amplitude ring $4, P=0.178$ ). Abnormal mfERG responses were found for all tested eccentricities, though effect size was clearly smaller for more peripheral rings.

No significant differences in implicit times were found for P1 peak time response between the two groups, for any of the eccentricity rings (Fig. 5C). No correlation was found between N1 or P1 peak amplitude and disease duration. Disease progression (duration and stage), however, was significantly correlated with P1 implicit time (Fig. 5D; disease duration $r=$ 0.212, $P=0.007$; disease stage $r=0.407, P<0.0001)$; therefore, delayed mfERGs responses may well appear in later stages of the disease. $\mathrm{P} 1$ grand average mfERG responses were highly correlated with CS measures $(r=0.491, P=0.037$ for the global CS-P1 sum correlation), especially for the more central retina locations (zone $0: r=0.508, P=0.030$; zone 1 : $r=0.490, P=0.038$ ) but not for the most peripheral area (zone 2: $r=0.262, P=0.299$ ). P1 amplitudes at ring 1 were also significantly correlated with global CS measures $(r=$ $0.514, P=0.028)$. Partial correlation analyses proved that the correlations of mfERG measures with CS function were independent of the correlations of the latter, with clinical parameters such as disease duration and visual acuity.

Morphologic Measures Obtained Using OCT and Correlation with Psychophysical Performance. OCT images acquired through the fovea showed three major patterns of retinal histopathology (Fig. 6): pattern A, relatively preserved central foveal depression and mild thickness increase of the retina; pattern $B$, severe thinning of neurosensory retina at the fovea and moderate elevation of the retina; pattern $\mathrm{C}$, severe thinning of neurosensory retina at the fovea and cystic macular changes in the outer retinal layers that can vary in size and number and that eventually span nearly the entire thickness of the retina. Five of 19 (26\%) eyes studied shared pattern A, had a mean central foveal thickness (ring 0 ) of $255 \pm 20 \mu \mathrm{m}$, and 
$\mathbf{A}$
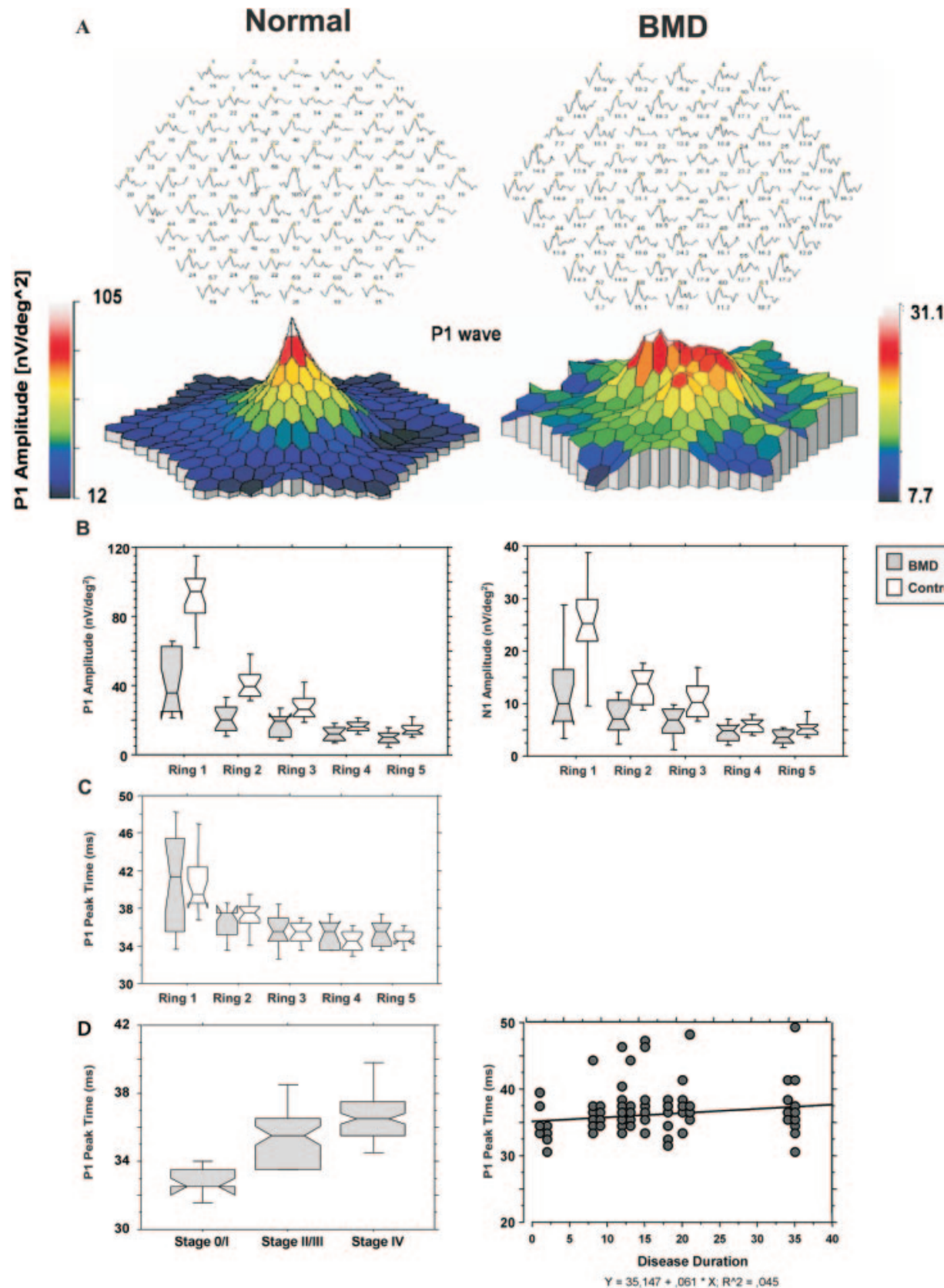

FiguRE 5. (A) mfERGs obtained from representative BMD patients and control subjects. Plots of the 61 hexagonal responses are presented. For each hexagonal response, the hexagon number (above the local ERG) and the respective $\mathrm{P} 1$ amplitude (below the local ERG) are indicated. Three-dimensional plots of P1 amplitudes obtained by mfERG clearly show higher amplitudes on the central hexagons than in the peripheral tested locations. (B) Statistical analyses of mfERG peak amplitudes are shown. P1 and N1 amplitudes were significantly reduced in BMD for different eccentricity rings up to $30^{\circ}$. (C) P1 peak time was not significantly altered between patients and controls for any of the rings examined. (D) Correlation of P1 peak time with disease progression (disease stage and duration). were characterized by a typically preserved VA (mean VA, 0.91 ) and short disease duration (mean, 11.9 years). Pattern $B$ was found in 3 of 19 (16\%) eyes with significantly reduced mean VA (mean VA, 0.67; $P<0.0001$ ) and longer disease duration (mean, 14.3 years; $P=0.587$ ) than those of pattern $A$. In pattern $\mathrm{C}$, where serous retinal detachment was observed, the mean VA was 0.80 , and retinal thickness was significantly higher for the two central rings than in pattern $\mathrm{B}(P=0.011$ for the central ring and $P=0.043$ for the after ring). The longest disease duration was observed in pattern C (20.8 years; $P=$ $0.024)$. Therefore, with disease progression, histologic profiles of BMD evolved from pattern A to pattern B, which turned out to be highly correlated with VA loss. Later, cystic macular changes varying in size and number may appear; this pattern was more prevalent with long-term disease progression.

When morphologic evaluation obtained by OCT was correlated with measures of retinal function, we did observe that evolution from pattern A to pattern $B$ is characterized by a significant global chromatic CS dysfunction $(P=0.012)$. Thickness of the neurosensory retina in ring 1 was significantly correlated with functional parameters such as P1 amplitude and with chromatic and achromatic CS measures (P1 amplitude of ring $1, r=0.562, P=0.014$; chromatic CS measures, $r=0.357, P=0.037$; achromatic global CS, $r=0.507, P=$ 0.044). Interestingly, retinal thickness at ring 1 , a region characterized by a high density of photoreceptor cells, also showed a strong correlation with VA $(r=0.149, P=0.012)$ and disease duration $(r=-0.242, P=0.047)$.

\section{Discussion}

The present study provides new insights into structure-function correlations at the level of the neurosensory retina in $\mathrm{BMD}$, including the involvement of the central and peripheral cone pathways, achromatic channels in vision, and their relationship with clinical markers of disease progression. 


\section{Pattern A}
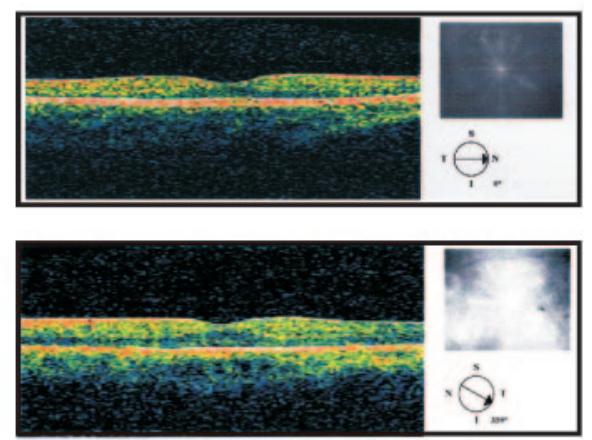

\section{Pattern B}
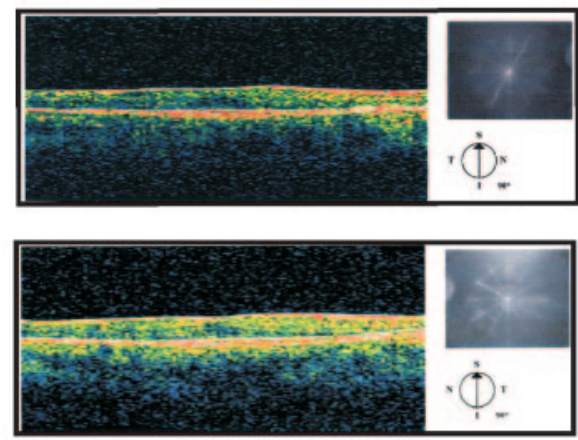

\section{Pattern C}
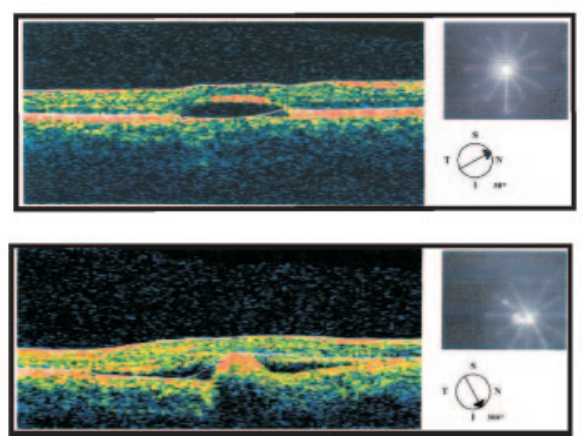

FIGURE 6. OCT images, representative of the three morphologic patterns found in BMD patients. Two examples per pattern are shown.

Accordingly, our work confirmed that BMD patients have neurosensory retina dysfunction up to $30^{\circ}$, as shown by reduced mfERG peak amplitudes and impaired peripheral achromatic CS. This functional impairment, which, according to Hood, ${ }^{27}$ can be speculated to be attributed to either cone photoreceptor cell loss or damage to the cone outer segments, was more extensive in our population sample than has been found by others. ${ }^{17,28-30}$ One explanation for this largely decreased mfERG response may be the larger number of affected eyes tested in the present study, which increases statistical power. On the other hand, this significantly reduced response in the periphery was also detected in adult-onset vitelliform macular dystrophy (AVMD), which has been suggested to have a considerable etiological overlap with Best disease. ${ }^{31,32}$ Our hypothesis that there is a pan-retinal defect in BMD is corroborated by the recently reported abundant expression of (mutated) bestrophin in the peripheral retina and of global retinal pigment epithelial failure (as obtained by the commonly altered EOG measures). ${ }^{1-3,33-35}$ We do believe that the extension of retinal damage and the familial intravariability and intervariability of age of onset and range of visual loss are part of a scenario of variable expression in BMD. ${ }^{36,37}$ Accordingly, for this autosomal recessive macular disease, there are reports of BMD families in whom some patients and generations are asymptomatic. $^{38,39}$ Thus, we speculate that the overlap between BMD and AVMD might be explained by the low penetrance and the great variability of disease symptoms in these vitelliform macular diseases.

The use of a new and comprehensive quantitative methodology to phenotype BMD provided interesting patterns of structure-function correlations. Morphologic alterations were functionally accompanied by correlated decreases in mfERG responses and reduced achromatic CS function with peripheral predominance. Furthermore, central deficits in the chromatic CS function were also typical as early indicators of functional damage, extending our previous work ${ }^{14}$ showing that macular S-cone dysfunction can be found at least as early as protan and deutan deficits. The accumulation of "yellow" pigment in BMD may partly influence the sensitivity of the blue cone pathway in the central region, though subjects may still use preserved macular regions to perform the color discrimination task. Rod intrusion is probably not a main contributor to the phenotype given that there are few rods in the central retina.

Our previous study ${ }^{14}$ emphasized the detection of colorcontrast deficits at isoluminance, whereas the present study emphasized similar quantal catches. It appears that the former approach may be more sensitive to detect dysfunction in chromatic pathways. Disease progression (as assessed by duration after the onset of initial clinical manifestations) was characterized by OCT-documented changes of the histologic integrity of the retina. Moreover, disease progression was also correlated with impairment of the CS function, particularly in more peripheral regions of the retina. The amplitudes of mfERG responses were found to be dramatically reduced in all subjects. Part of the variance of these measures was highly correlated with CS performance, suggesting that mfERG and achromatic CS can be useful independent tools to evaluate early functional loss in members of families with suspected disease. It is important to emphasize that the robust central mfERG responses were obtained from BMD patients with relatively preserved VA.

Our study shows that cross-correlation analysis of electrophysiological, psychophysical, and morphometric data improve quantitative phenotyping in BMD. All our quantitative parameters showed significant correlations with clinical, psychophysical, and electrophysiological data. Furthermore, we suggest that achromatic CS and mfERG measures are especially important for detecting peripheral visual impairment early in families with a positive history of BMD. On the other hand, damage assessment in chromatic pathways, OCT morphologic evaluation, and central achromatic CS functions represent suitable approaches to study macular function and to complement the evaluation of damage and disease progression in BMD.

\section{Acknowledgments}

The authors thank the patients for their participation. They also thank Ana Rita Vaz, Mário Soares, and Pedro Melo for technical assistance.

\section{References}

1. Best F. Über eine hereditäre maculaaffektion: Beitäge zur Verebungslehre. Z Augenbeikd. 1905;13:199-212.

2. O'Gorman S, Flaherty WA, Fishman GA, Berson EL. Histopathologic findings in Best's vitelliform macular dystrophy. Arch $O p h$ thalmol. 1988;106:1261-1268.

3. Maloney WF, Robertson DM, Duboff SM. Hereditary vitelliform macular degeneration: variable findings within a single pedigree. Arch Opbthalmol. 1977;95:979-983. 
4. Mohler CW, Fine SL. Long-term evaluation with Best's vitelliform dystrophy. Ophthalmology. 1981;88:688-692.

5. Clemett R. Vitelliform dystrophy: long-term observations on New Zealand pedigrees. Aust N Z J Ophthalmol. 1991;19:221-227.

6. Marquardt A, Stohr H, Passmore LA, Kramer F, Rivera A, Weber BH. Mutations in a novel gene, $V M D 2$, encoding a protein of unknown properties cause juvenile-onset macular dystrophy (Best's disease). Hum Mol Genet. 1998;7:1517-1525.

7. Petrukhin K, Koisti MJ, Bakall B, et al. Identification of the gene responsible for Best macular dystrophy. Nat Genet. 1998;19:241247.

8. Marmorstein AD, Marmorstein LY, Rayborn M, Wang X, Hollyfield JG, Petrukhin K. Bestrophin, the product of the Best vitelliform macular dystrophy gene (VMD2), localizes to the basolateral plasma membrane of the retinal pigment epithelium. Proc Natl Acad Sci U S A. 2000;97:12758-12763.

9. Sun H, Tsunenari T, Yau KW, Nathans J. The vitelliform macular dystrophy protein defines a new family of chloride channels. Proc Natl Acad Sci U S A. 2002;99:4008-4013.

10. Marmorstein $\mathrm{AD}$, Stanton JB, Yocom $\mathrm{J}$, et al. A model of Best vitelliform macular dystrophy in rats. Invest Ophthalmol Vis Sci. 2004; $45: 3733-3739$

11. Weingeist TA, Kobrin JL, Watzke RC. Histopathology of Best's macular dystrophy. Arch Ophthalmol. 1982;100:1108-1114.

12. Frangieh GT, Green WR, Fine SL. A histopathologic study of Best's macular dystrophy. Arch of Ophthalmology. 1982;100:1115-1121.

13. Massof RW, Fleischman JA, Fine SL, Yoder F. Flicker fusion thresholds in Best macular dystrophy. Arch Ophthalmol. 1977;95:991994.

14. Campos SH, Forjaz V, Kozak LR, Silva E, Castelo-Branco M. Quantitative phenotyping of chromatic dysfunction in Best macular dystrophy. Arch Ophthalmol. 2005;123:944-949.

15. Scholl HP, Kremers J, Apfelstedt-Stylla E, Zrenner E. L- and M-cone driven ERGs are differently altered in Best's macular dystrophy. Vision Res. 2000;40:3159-3168.

16. Falsini B, Porciatti V, Porrelo G, et al. Macular flicker electroretinograms in Best vitelliform dystrophy. Curr Eye Res. 1996;15:638646.

17. Scholl HP, Schuster AM, Vonthein R, Zrenner E. Mapping of retinal function in Best macular dystrophy using multifocal electroretinography. Vision Res. 2002;42:1053-1061.

18. Caprioli J. Automated perimetry in glaucoma. Am J Ophthalmol. 1991;111:235-239.

19. Regan BC, Reffin JP, Mollon JD. Luminance noise and the rapid determination of discrimination ellipses in color deficiency. Vision Res. 1994;34:1279-1299.

20. Kremers J, Maciej WS, Scholl HP, Saito C. Cone selective adaptation influences L- and M-cone drives signals in electroretinography and psychophysics. J Vision. 2003;3:146-160.

21. Albrecht J, Jagle H, Hood DC, Sharpe LT. The multifocal electroretinogran (mfERG) and cone isolating stimuli: variation in L- and M-cone drives signals across the retina. J Vision. 2002;2:543-558.
22. Wyszecki G, Stiles WS. Color Science: Concepts and Methods, Quantitative Data. 2nd ed. New York: John Wiley \& Sons; 1982.

23. Pugh EN. Vision: physics and retinal physiology. In: Atkinson RC, ed. Stevens Handbook of Experimental Psychology. New York: John Wiley \& Sons; 1988.

24. Kutschbach E. Method for Multifocal ERG Using Short Length and Corrected M-Sequences. Wiesbaden: Roland Consult Elektrophysiologische Diagnostik Systeme; 1997.

25. Hood DC, Seiple W, Holopigian K, Greenstein V. A comparison of the components of the multifocal and full-field ERGs. Vis Neurosci. 1997; 14:533-544.

26. Brancato R, Lumbroso B. Guide to Optical Coherence Tomography Interpretation. Rome: Innovation-News-Communication; 2004.

27. Hood DC. Assessing retinal function with the multifocal technique. Prog Retinal Eye Res. 2000;19:607-646.

28. Schatz P, Klar J, Andreasson S, Ponjavic V, Dahl N. Variant phenotype of Best vitelliform macular dystrophy associated with compound heterozygous mutations in VMD2. Ophthalmic Genet. 2006;27:51-56

29. Palmowski AM, Allgayer R, Heinemann-Verbaleken B, Scherer V, Ruprecht KW. Detection of retinal dysfunction in vitelliform macular dystrophy and the multifocal ERG (mf-ERG). Doc Ophthalmol. 2003;106:145-152

30. Glybina IV, Frank RN. Localization of multifocal electroretinogram abnormalities to the lesion site: findings in a family with Best disease. Arch Ophthalmol. 2006;124:1593-1600.

31. Saito W, Yamamoto S, Hayashi M, Ogata K. Morphological and functional analyses of adult onset vitelliform macular dystrophy. Br J Ophthalmol. 2003;87:758-762.

32. Pierro L, Tremolada G, Introini U, Calori G, Brancato R. Optical coherence tomography findings in adult-onset foveomacular vitelliform dystrophy. Am J Ophthalmol. 2002;134:675-680.

33. Mullins RF, Kuehn MH, Faidley EA, Syed NA, Stone EM. Differential macular and peripheral expression of bestrophin in human eyes and its implication for Best disease. Invest Ophthalmol Vis Sci. 2007; 48:3372-3380.

34. Marmor MF, Zrenner E. Standard for clinical electro-oculography. Doc Ophthalmol. 1993;85:115-124.

35. Seddon JM, Sharma S, Chong S, Hutchinson S, Allikmets R, Adelmar RA. Phenotype and genotype correlations in two Best families. Ophthalmology. 2003;110:1724-1731.

36. Renner AB, Tillack $\mathrm{H}$, Kraus $\mathrm{H}$, et al. Late onset is common in best macular dystrophy associated with VMD2 gene mutations. Ophthalmology. 2005;112:586-592.

37. Mullins R, Oh K, Heffron E, Hageman G, Stone E. Late development of vitelliform lesions and flecks in a patient with Best's disease: clinicopathologic correlation. Arch Ophthalmol. 2005; 123:1588-1594.

38. Weber BHF, Walker D, Müller B. Molecular evidence for nonpenetrance in Best's disease. J Med Genet. 1994;31:388-392.

39. Nordström S. Variable expression of tapetoretinal degeneration in northern Sweden. Hereditas. 1993;118:15-19. 